\title{
Nondiffracting Waves in Anisotropic Media
}

\author{
J. Salo and M. M. Salomaa \\ Materials Physics Laboratory, Helsinki University of Technology \\ P. O. Box 2200 (Technical Physics), FIN-02015 HUT, Finland
}

\begin{abstract}
Recently, the physics of ballistic phonon propagation in anisotropic crystals has been studied with new phonon-imaging methods. In this paper we consider nondiffracting waves that can propagate in anisotropic crystals and analyze their properties that emerge specifically due to the anisotropy. We further present a detailed generation and detection scheme for the experimental verification of the wave modes considered.
\end{abstract}

PACS numbers: $43.20 .+\mathrm{g}, 46.05 .+\mathrm{b}, 46.40 . \mathrm{Cd}, 62.30 .+\mathrm{d}$

\section{INTRODUCTION}

Ballistic propagation of phonons in crystals, governed by the (piezo-)elastic equations of motion, has lately been extensively investigated with the help of methods of imaging acoustic phonon propagation in solids [1]. The underlying anisotropic crystal has a strong influence on the propagation of lattice vibrations, associated both with the heat flux and with the acoustic (sound) waves. The formation of wave fronts due to phonon focusing clearly differs from that in isotropic media, leading to effects like caustics and internal diffraction. These may be understood in terms of the slowness- and groupvelocity surfaces characteristic to each individual crystal; see, e.g., Refs. [2].

Here we consider nondiffracting waves (NDWs), i.e., propagation-invariant waves - originally discovered in optics by Durnin et al. in 1987 [3] — that propagate in crystalline solids [4]. They are not simply wave fronts emanating from abrupt heat pulses, but, instead, they feature a unique phase velocity along their propagation direction. Consequently, their different wave components remain in phase and the entire shape of the wave pattern remains invariant under propagation. Nondiffracting waves may be of two different kinds, either (i) continuous Bessel-beam like waves with unique temporal frequency, $\omega$; or (ii) pulsed propagating waves, i.e., the socalled $\mathrm{X}$ waves $[5,6]$.

While isotropic nondiffracting waves are adequately described with closed-form expressions, their anisotropic counterparts only have integral representations. Here we also derive an approximate asymptotic representation for anisotropic NDWs that allows a physical interpretation in terms of energy propagation associated with the waves. Although the developed theory is only valid in regions away from the axis of propagation, the generation of NDWs is naturally described using this scheme.

These results are then applied in two different contexts: Firstly, (i) We discuss effects specially related to the anisotropy. The intensities of the nondiffracting wave fields along different radial directions are shown to depend on the curvature of the slowness curve while caustics [7] and internal diffraction [8] effects are due to its local nonconvexity. We also demonstrate that the walkoff effect may under certain conditions prohibit the propagation of nondiffracting waves since the group velocity of the individual wave components may sometimes point in the opposite direction relative to that of beam propagation.

Secondly, (ii) We use the asymptotic representation to design a transducer arrangement on a piezoelectric crystal that can be used to generate a nondiffracting wave field within the crystal. Aperture optimization is also discussed, based on the propagation directions of the individual wave components. We anticipate that this experimental setup would serve to verify the theoretical results.

\section{ANISOTROPIC NONDIFFRACTING WAVES}

Both low-frequency acoustic waves and high-frequency heat-flow phonons obey the elastic equations of motion, characterized by the stiffness tensor of the crystal. These equations are nondispersive in the long-wavelength limit, where the wavelength $\lambda$ is much larger than the average interatomic separation. For the general theory of elasticity and piezoelectricity, see, for instance, Refs. [1, 9, 10].

Plane waves in elastic piezoelectric media,

$$
\mathbf{u}(\mathbf{r} ; t)=\mathbf{U} e^{i(\mathbf{k} \cdot \mathbf{r}-\omega t)},
$$

obey the Christoffel (eigenvalue) equation

$$
\sum_{l, m, n=1}^{3} \tilde{c}_{k l m n} k_{l} k_{n} U_{m}=\rho \omega^{2} U_{k}
$$

for each $k=1,2,3$. Here $\tilde{c}_{k l m n}$ is the tensor of piezoelectrically stiffened elastic moduli, cf. Ref. [10]. 
In elastic solids (and piezoelectric crystals), there exist in general three propagating plane-wave modes along each spatial direction. These modes have orthogonal polarizations $\mathbf{U}$ (i.e., directions of displacement) and they often propagate with different phase velocities. These plane-wave modes are characterized with their slowness vectors, $\mathbf{s}=\mathbf{k} / \omega$ that point in the direction of the wave vector and have the magnitude of inverse phase velocity. The slownesses of the three plane-wave modes trace three closed slowness surfaces when the direction of the wave vector spans over all spatial directions.

A wave field $\mathbf{u}(\mathbf{r} ; t)$ in an infinite homogeneous medium is nondiffracting provided that it propagates uniformly, i.e., invariant in shape along, say, the $z$-coordinate,

$$
\mathbf{u}(\mathbf{r} ; t)=\mathbf{u}(x, y, z-v t) .
$$

The velocity of propagation $v$ is a free parameter that may be chosen independently of the phase (or group) velocity in the medium or of the direction of propagation [11]. The vector field $\mathbf{u}$ may comprise, in addition to the mechanical displacements $U_{i}$, several other components, such as electrostatic (magnetostatic) potential (in the quasi-static approximation), and electromagnetic field components (in the ultra-high frequency range). The spatio-temporal Fourier transform of a nondiffracting wave, Eq. (3), proves to be proportional to $\delta\left(k_{z}-\omega / v\right)$ which is both a sufficient and a necessary condition for the existence of nondiffracting waves $[4,12]$.

\section{A Integral representation of NDWs}

Nondiffracting waves are superpositions of plane waves that all satisfy the common condition $k_{z}=\omega / v$. Hence all the wave components remain in phase while propagating along $z$ without any alteration in the wave pattern. This condition is satisfied if all the wave components have the same common (phase) slowness along $z$, namely $s_{z}=1 / v$ (note that the orientation of the crystal axes may be arbitrary and the $z$ axis only refers to the desired direction of nondiffracting wave propagation). Therefore, nondiffracting modes are characterized by the intersection of the slowness surfaces with the $s_{z}=1 / v$ plane, see Fig. 1. The intersection contains at most three slowness curves, each of which may be used to construct a separate class of nondiffracting waves. Note that these slowness curves need not be symmetric with respect to the $s_{z}$ axis since they do not lie in a plane passing through the origin.

The general form of nondiffracting waves is obtained as the superposition of propagating plane waves [13] that obey the nondiffraction condition, Eq. (3),

$$
\mathbf{u}=\iint A(\omega, \theta) \mathbf{U}(\theta) e^{i \omega[\mathbf{s}(\theta) \cdot \mathbf{r}-t]} d \theta d \omega .
$$

Here the $\theta$-integral extends over one or more slowness curves in the $s_{z}=1 / v$-cut plane, and the $\omega$ integration (a)
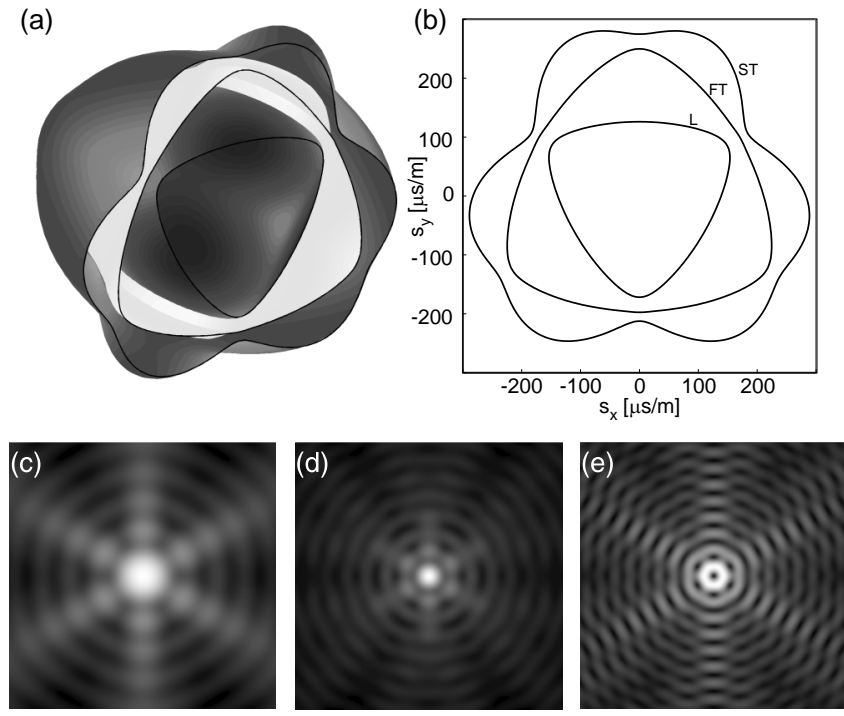

0

1

FIG. 1: Three nondiffracting wave modes in quartz. (a) Three slowness surfaces cut off at $s_{z}=70 \cdot 10^{-6} \mathrm{~s} / \mathrm{m}$, corresponding to the phase velocity $v=14286 \mathrm{~m} / \mathrm{s}$. (b) Slowness curves on the $\left(s_{x}, s_{y}\right)$-plane. (c) Longitudinal (L), (d) fast transverse (FT), and (e) slow transverse (ST) beam modes. All beams are fundamental modes with the angular frequency $\omega=10^{9} \mathrm{~s}^{-1}$. Areas illustrated are $200 \mu \mathrm{m} \times 200 \mu \mathrm{m}$ and the color shading denotes the time-averaged kinetic energy.

covers different frequency components. The arbitrary function $A(\omega, \theta)$ represents the weight of each plane-wave component. We choose $\theta$ as the arc-length parametrization of the slowness curve(s) (see Section III); all other contributions are included in the weight function $A(\omega, \theta)$. The polarization is only defined up to a complex constant factor by the Christoffel Eq. (2), and it should be chosen continuous along the given slowness curve, i.e., the integration path.

Provided that the weight function is assumed separable: $A(\omega, \theta)=f(\omega) \beta(\theta)$, there are two ways to considerably simplify the wave expression: If the spectral part $f(\omega)$ can be integrated analytically, the frequency integral is performed first. In particular, X-wave-type spectra $f(\omega)=\omega^{m} e^{-\alpha \omega}$ yield

$$
\int_{0}^{\infty} \omega^{m} e^{-\alpha \omega} e^{i \omega(\mathbf{s}(\theta) \cdot \mathbf{r}-t)} d \omega=\frac{m !}{\{\alpha-i[\mathbf{s}(\theta) \cdot \mathbf{r}-t]\}^{m+1}}
$$

while the entire pulse is given by

$$
\mathbf{u}=\int \frac{m ! \beta(\theta) \mathbf{U}(\theta)}{\{\alpha-i[\mathbf{s}(\theta) \cdot \mathbf{r}-t]\}^{m+1}} d \theta .
$$

If the spectral integral cannot be evaluated analytically, the pulse can be obtained by first numerically finding the 

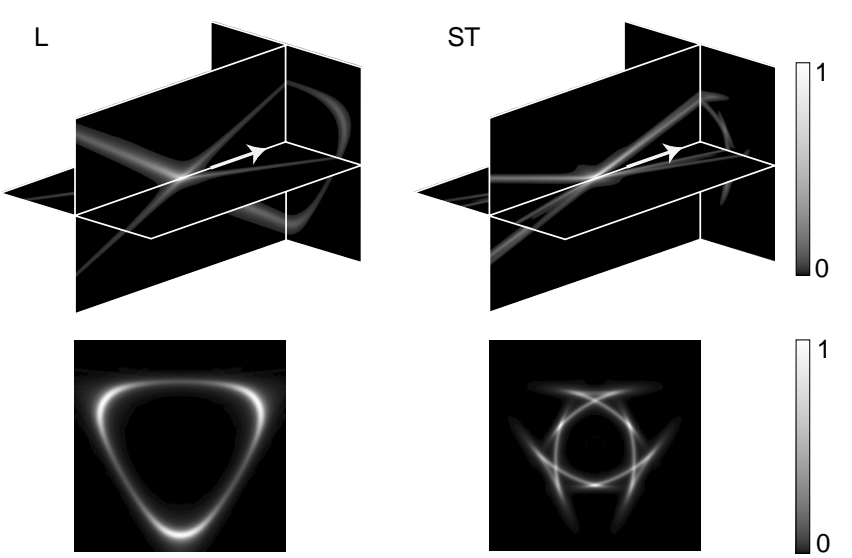

FIG. 2: (Top) Longitudinal (L) and slow transverse (ST) pulse mode in quartz. (Bottom) Their approaching cross sections (back planes in top, $z>v t$ ). Both pulses correspond to the slowness cuts in Fig. 1. White arrows denote the propagation of the pulse pattern. The cross sections are directly obtained from the radial-velocity curves in Fig. 4.

monochromatic beam

$$
\mathbf{v}(\mathbf{r}, t ; \omega)=\int \beta(\theta) \mathbf{U}(\theta) e^{i \omega\left[s_{x}(\theta) x+s_{y}(\theta) y+s_{z} z-t\right]} d \theta
$$

that can further be integrated over $\omega$ in order to obtain the anisotropic pulse

$$
\mathbf{u}=\int f(\omega) \mathbf{v}(\mathbf{r}, t ; \omega) d \omega
$$

If the constant-frequency wave is evaluated for a reference frequency $\omega^{\prime}$, all the other frequencies may be obtained simply by scaling

$$
\mathbf{v}(\mathbf{r}, t ; \omega)=\mathbf{v}\left(\frac{\omega}{\omega^{\prime}} \mathbf{r}, \frac{\omega}{\omega^{\prime}} t ; \omega^{\prime}\right)
$$

and the nondiffracting pulses can be represented as

$$
\mathbf{u}=\int f(\omega) \mathbf{v}\left(\frac{\omega}{\omega^{\prime}} \mathbf{r}, \frac{\omega}{\omega^{\prime}} t ; \omega^{\prime}\right) d \omega
$$

The structure of the resulting wave is determined by the shape function $\beta(\theta)$, and each frequency component has the same spatial shape, only scaled by the frequency.

The entire information on the governing wave equation is contained in the plane-wave solutions and the nondiffraction property is guaranteed by confining onto the $s_{z}=1 / v$-cut plane of the slowness surfaces. The remaining freedom is contained in the weight function $A(\omega, \theta)$. We refer to the special case of $\beta(\theta) \equiv 1$ as the fundamental mode. For illustrations of fundamental beam modes, see Fig. (1) and for those of the corresponding pulses, Fig. (2).

\section{B Asymptotic form of the wave field}

Although the integrals in Eq. (4) are readily evaluated numerically once the slowness curves are known, the computational results offer little direct physical insight into the nature of the nondiffracting waves. Therefore, we rather derive an asymptotic evaluation of the integrand of Eq. (7). Using cylindrical coordinates, $\mathbf{r}=\left(r_{\perp}, \varphi, z\right)$, the nondiffracting beam assumes the form

$$
\mathbf{v}(\mathbf{r}, t ; \omega)=e^{i \omega\left[s_{z} z-t\right]} \int \beta(\theta) \mathbf{U}(\theta) e^{i r_{\perp} \omega \hat{\mathbf{r}}_{\perp} \cdot \mathbf{s}(\theta)} d \theta,
$$

where $\hat{\mathbf{r}}_{\perp}=\hat{\mathbf{i}} \cos \varphi+\hat{\mathbf{j}} \cos \varphi$ is the radial unit vector. For large enough $r_{\perp} \omega$, the exponential term in the integral in Eq. (11) oscillates rapidly, and the integral tends to zero (assuming that $\beta(\theta) \mathbf{U}(\theta)$ is sufficiently smooth). Note that $\beta$ and $\mathbf{U}$ never compensate these oscillations since they are independent of both frequency and spatial coordinates. In the asymptotic evaluation of the integral, we look for the leading terms that decay comparatively slowest for large $r$. The solution is derived in the Appendix; it allows for the following interpretation:

The main contribution to the integral for a fixed spatial direction $\varphi$ arises from those points on the slowness curve where the normal of the curve is collinear with the chosen direction. This is, in fact, reminiscent of the general property in anisotropic wave motion: the direction of energy propagation is along the normal of the slowness surface, and the dominant contribution is due to those components that transport energy in the direction of observation.

The asymptotic contributions to the wave are expressed as

$$
\begin{aligned}
& \mathbf{v}(\mathbf{r}, t ; \omega) \\
& \approx \sum_{k: \mathbf{n}_{k} \| \mathbf{r}_{\perp}} \sqrt{\frac{2 \pi}{\omega r_{\perp} \kappa\left(\theta_{k}\right)}} \beta\left(\theta_{k}\right) \mathbf{U}\left(\theta_{k}\right) e^{ \pm i \pi / 4} e^{i \omega\left[\mathbf{s}\left(\theta_{k}\right) \cdot \mathbf{r}-t\right]}
\end{aligned}
$$

where the summation is taken over the points specified above and $\kappa(\theta)=\left|\mathbf{s}^{\prime \prime}(\theta)\right|$ is the curvature of the slowness curve. The \pm refers to the sign of $\mathbf{r}_{\perp} \cdot \mathbf{s}_{\perp}^{\prime \prime}\left(\theta_{k}\right)$ in the exponential term. It is negative for waves emanating from the axis while it has a positive value for waves propagating towards the axis. Each term in the wave expression is a plane wave arriving to the beam axis from the direction $\varphi$ and, together, they constitute a generalized conical wave. It may, however, have a complicated folded form as will be discussed below in Section III.

The above expression, Eq. (12), is valid for all directions where the denominator does not vanish, which would cause the expression to diverge. Although such divergences only occur in the asymptotic expression (the original integral never diverges), they imply the existence of a caustic associated with a flat point in the slowness surface, see Subsection III B below. The approximation 

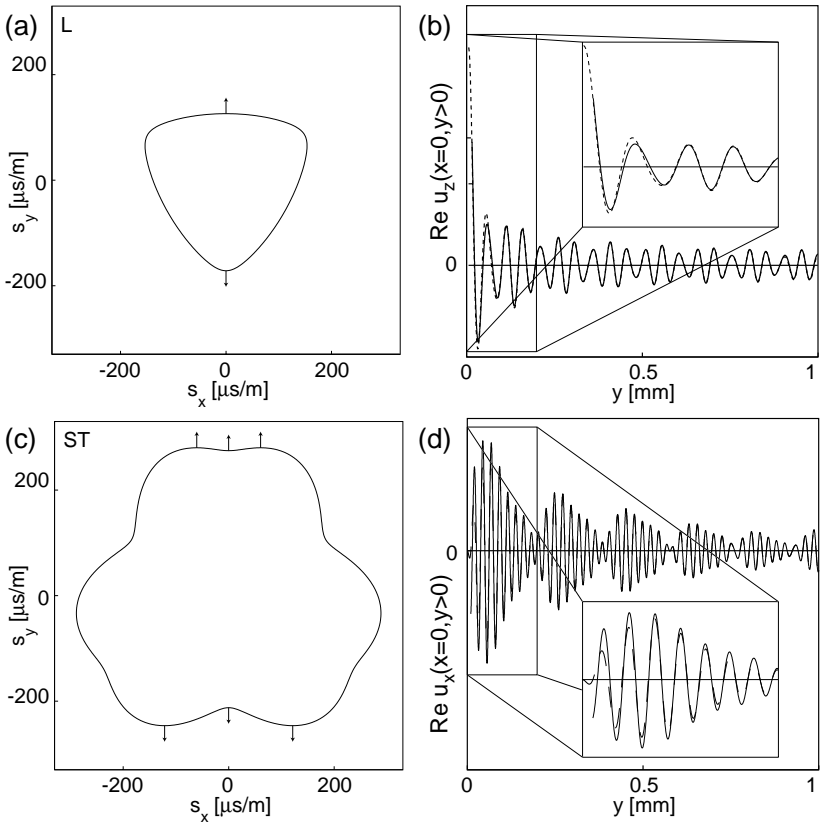

FIG. 3: Convergence of the asymptotic approximation along $y$. (a) The slowness curve of the longitudinal (L) wave, (b) numerical integral (dashed line) and asymptotic (solid line) solutions for $u_{z}$, (c) slowness curve for the slow transverse (ST) wave, and (d) integral (dashed line) and asymptotic (solid line) solutions for $u_{x}$. Waves represent the fundamental mode with $\beta(\theta) \equiv 1$. The dominant wave components are denoted with the outer normals to the slowness curves.

converges rapidly for a slowly varying shape function $\beta(\theta)$ (especially for the fundamental mode), see Fig. 3. However, in the case that either $\beta$ or the polarization changes rapidly along the slowness curve, convergence is only achieved for large $r$.

The asymptotic form for the nondiffracting pulse is obtained from Eq. (8) together with the asymptotic nondiffracting beam, Eq. (12) above. Choosing an $\mathrm{X}$ wave spectrum $f(\omega)=\omega^{m} e^{-\alpha \omega}$ and using the integration result $[14]$

$$
\int_{0}^{\infty} \frac{\omega^{m}}{\sqrt{\omega}} e^{-\alpha \omega} d \omega=\frac{\Gamma\left(m+\frac{1}{2}\right)}{\alpha^{m+1 / 2}},
$$

the wave assumes the analytic form

$$
\begin{aligned}
\mathbf{u}_{m}(\mathbf{r}, t) \approx \sum_{k} & \sqrt{\frac{2 \pi}{r_{\perp} \kappa\left(\theta_{k}\right)}} \beta\left(\theta_{k}\right) \mathbf{U}\left(\theta_{k}\right) e^{ \pm i \pi / 4} \\
& \times \frac{\Gamma\left(m+\frac{1}{2}\right)}{\left\{\alpha-i\left[\mathbf{s}\left(\theta_{k}\right) \cdot \mathbf{r}-t\right]\right\}^{m+1 / 2}} .
\end{aligned}
$$

Although indicative, this result should not be taken literally under the actual physical circumstances: The asymptotic form, Eq. (12), converges for large $\omega r_{\perp}$ and, thus, the low-frequency spectrum in the frequency domain (near zero) is invalid for arbitrary $r_{\perp}$. However, for large values of $m$ the dominant frequencies are high and the approximation is fair for $r$ in the pulse cone and beyond. Nonetheless, asymptotics of the transverse wave pattern may be explained with the use of this approximation.

\section{EFFECTS DUE TO ANISOTROPY}

Nondiffracting waves in isotropic media feature a characteristic cone of propagation $[6,12]$ that asymptotically describes the wave propagation. For nondiffracting beams, the cone forms surfaces of equal phase and energy flows along the normal to the cone. Furthermore, the energy of the propagating waves is equally distributed on the cone of constant $|\beta(\theta)|$. As for nondiffracting pulses, the cone forms the actual propagating wave fronts. Note that, in practice, both the phase and the energy intensity depend on the shape function $\beta(\theta)$ but not on the underlying physics which in isotropic media gives no 'preferred' directions of energy propagation.

In this Section, we use the asymptotic wave solution to generalize the cone of propagation to anisotropic nondiffracting waves, and to derive the 'natural' energy distribution thereon.

\section{A Anisotropic wave fronts}

The maximal amplitude of individual wave components occurs at the minimum of the denominator in Eq. (14). The radial vectors pointing to the maxima are given by

$$
\mathbf{R}_{\perp}=\frac{t-s_{z} z}{\mathbf{s}_{\perp} \cdot \mathbf{n}_{\perp}} \mathbf{n}_{\perp}=\frac{t-z / v}{\mathbf{s}_{\perp} \cdot \mathbf{n}_{\perp}} \mathbf{n}_{\perp}
$$

for fixed $z$ and $t$. Here, the $\mathbf{s}_{\perp}$ are radial slowness vectors and $\mathbf{n}_{\perp}$ are the (radial) normals to the slowness curve. The cross-sectional shape of the nondiffracting pulse is obtained by allowing the slowness to vary along the associated slowness curve, see Fig. 4.

For constant $z$, the field maxima first approach the axis of propagation and, once having crossed it, they continue their propagation outwards toward infinity. The radial velocity of the field maxima is given by

$$
\mathbf{V}_{\perp}=\frac{\mathrm{d} \mathbf{R}_{\perp}}{\mathrm{d} t}=\frac{\mathbf{n}_{\perp}}{\mathbf{s}_{\perp} \cdot \mathbf{n}_{\perp}}
$$

and it satisfies the group-velocity-like relation $\mathbf{V}_{\perp} \cdot \mathbf{s}_{\perp}=$ 1. Note that the scalar product is only taken for the radial components of the vectors. The radial velocity of the field is not the radial component of group velocity since the latter obeys $\mathbf{V}_{g} \cdot \mathbf{s}=\mathbf{V}_{g \perp} \cdot \mathbf{s}_{\perp}+V_{z} s_{z}=1$ and since, in general, $V_{z} s_{z} \neq 0$ the radial part of the inner product cannot equal unity for the group velocity. 


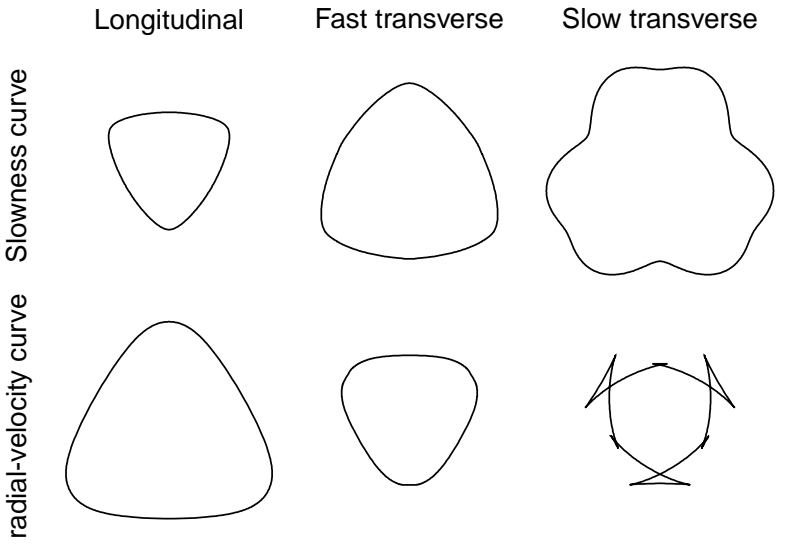

FIG. 4: Three slowness curves and the corresponding radial-velocity curves for quartz. Only the ST mode features nonconvex slowness and, consequently, its velocity curve exhibits folds associated with wave-front caustics. The radial-velocity curves readily provide the drifting cross sections $(z<v t)$; the approaching cross sections in Fig. 2 are obtained after inversion $(x \rightarrow-x, y \rightarrow-y)$.

The beam amplitude of the asymptotic expression, Eq. (12), depends on the following two factors: (i) the curvature, $\kappa$, of the slowness curve, and (ii) the shape function, $\beta$. The former is purely characteristic to the medium and the direction and velocity of propagation (which together serve to define the slowness curve) while the latter describes the excitation of the wave in the Fourier domain. For isotropic wave motion, curvature is constant and the amplitude depends solely on the shape function.

\section{B Caustics and internal diffraction}

The wave amplitude is proportional to $|\beta(\theta)| / \sqrt{\kappa(\theta)}$ and, hence, a small curvature of the slowness (i.e., large radius of curvature) implies an elevated level of wave amplitude. This is called phonon focusing. If, however, the slowness curve is flat, i.e., its curvature vanishes, the asymptotic expansion based on the stationary-phase approximation no longer holds. This is due to the fact that all the wave components in the vicinity of the zerocurvature point propagate into the same direction, causing an increase in the field amplitude, or a caustic.

Another consequence of vanishing curvature is the appearance of internal diffraction. At a caustic point, the number of contributing wave components changes abruptly. Normally, the curvature of the slowness is negative (meaning that the scalar product between the slowness vector and its second derivative is negative). At the caustic points, however, the curvature changes sign; this is observed in two ways: (i) the slowness curve is no longer convex; and (ii) the radial-velocity curve develops
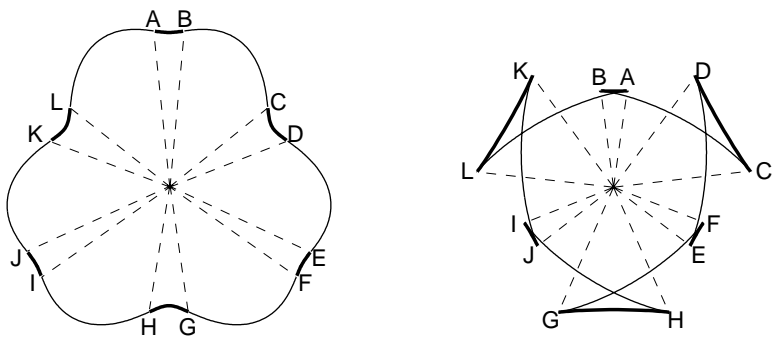

FIG. 5: Caustics of the slow transverse mode in quartz. The zero-curvature points on the slowness surface (lhs) imply caustics which are observed as folds in the velocity curve (rhs). Thick lines denote areas of positive curvature and capital letters indicate the individual caustics.
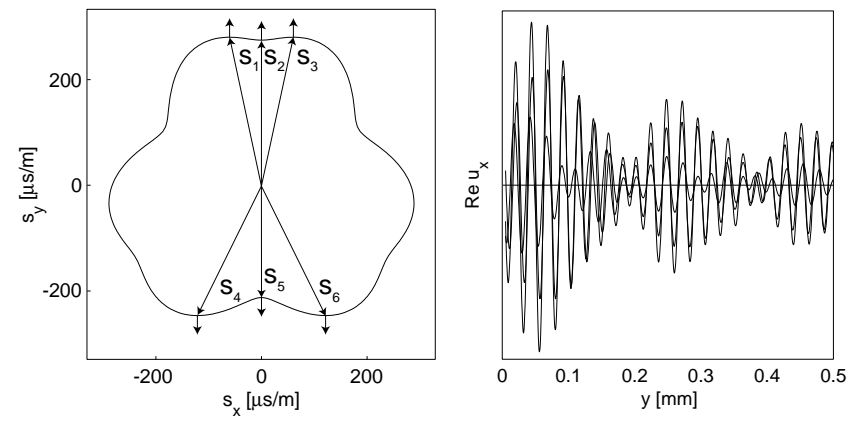

FIG. 6: Internal diffraction causes an interference pattern between several wave modes that propagate along the same radial direction. For the case illustrated here, the interference period of approximately $0.19 \mathrm{~mm}$ is due to the difference between the waves $\mathbf{s}_{1,3}$ and $\mathbf{s}_{4,6}$ which, together, carry most of the wave energy along $y$. The interfering wave vectors are $k_{y, 1,3}=246 \mathrm{~mm}^{-1}$ and $k_{y, 2,4}=$ $280 \mathrm{~mm}^{-1}$ for the angular frequency $\omega=10^{9} \mathrm{~s}^{-1}$.

folds, implying that several wave modes propagate along the same radial direction, see Fig. 5.

Internal diffraction is sometimes observed as a clear interference pattern of the participating wave components, see Fig. 6. This is due to the fact that, for a singlefrequency beam, there are from 1 to 6 plane waves with different wave numbers and amplitudes that propagate along the same direction. For a pulse, this leads to a folded cone, and if the cone is thick enough, the different folds may overlap, leading to mutual interference. This effect has been observed for normal wave fronts in crystals, see Ref. [1].

\section{Energy propagation}

Apart from the factor $r_{\perp}^{-1 / 2}$, the asymptotic expression, Eq. (12), consists of plane waves whose directions 
of energy transport are given by the corresponding groupvelocity vectors, V. In isotropic materials, the group velocity is always collinear with the wave-front propagation, while in nondispersive materials the group velocity along the wave-front propagation direction equals the phase velocity. Now the latter holds but the group velocity may also contain a transverse component with respect to the wave front. In the asymptotic region of nondiffracting waves, the group velocity has no azimuthal component since it is normal to the slowness curve. Hence, the energy flow in the asymptotic range only has a radial $(r)$ and an axial $(z)$ component.

The nondiffracting-wave 'cone', which constitutes the pulse itself, is essentially formed by plane waves, although different ones in the different directions. The energy flow associated with these plane waves does not necessarily propagate along positive $z$, see Fig. 7 . If the pulse is to be generated at $z=0$ and it should propagate along positive $z$, all the group velocities must also posses a positive component along $z$. This is readily fulfilled for the longitudinal mode in Fig. 7 but not for the slow transverse mode.

\section{ACOUSTIC GENERATION OF NDWS}

In this Section, we consider a method for the experimental generation of NDWs into elastic crystals. Within optics, there are several methods for producing nondiffracting waves. Durnin et al. used a circular slit followed by a Fourier-transforming lens [3]. Axicons [15] have also been used to produce Bessel beams, first by Bunkin et al. [16] (already before Durnin's work), and later by Scott and McArdle [17], and others. Possibly the most adjustable method for generating NDWs is the use of computer-generated holograms, i.e., diffractive elements [18]. We propose a similar arrangement, consisting of a piezoelectric transducer fabricated on the top surface of a bulk crystal.

\section{A Transducer arrangement}

We consider the design of a piezoelectric transducer operating in the asymptotic region of a nondiffracting beam. Taken a beam that has no regions of internal diffraction, i.e., its radial-velocity curve has no folds and there is only one wave mode along each direction that moves towards the axis. For simplicity, we take this radial direction as the positive $x$ axis.

Thus, the plane wave has $s_{z}=1 / v, V_{\perp x}<0$, and $V_{\perp y}=0$. Both $s_{x}$ and $s_{y}$ are obtained uniquely from the asymptotic field solution. The field on the substrate surface exhibits a periodicity determined by the wave vector $\left(k_{x, 0}, k_{y, 0}\right)=\left(\omega s_{x}, \omega s_{y}\right)$ and, therefore, the transducer must also have the same period. However, the realization of a continuous-profile transducer that is described

\section{0}
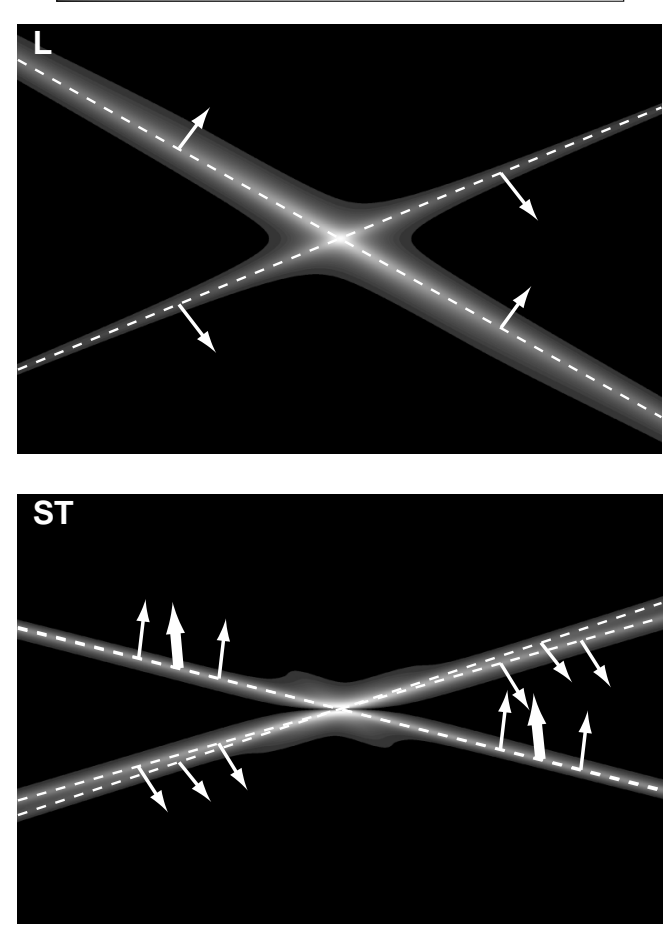

FIG. 7: Nondiffracting pulses of L and ST modes in the meridional $(y, z)$ plane. Wave energy in anisotropic crystals does not propagate perpendicular to the wave fronts. While in the L mode (top) the group velocities of both wave components have positive velocity along $z$, this does not hold for the ST mode (bottom). Although the wave pattern in the latter propagates along positive $z$, part of the wave energy is transported into the opposite direction. Hence, the wave may not be launched from a transducer placed at $z=0$. In the ST mode, there are actually six modes present, the same as indicated in Fig. 3. Here the negative $V_{z}$ component is denoted with bold arrows.

by one single wave vector is practically impossible and the structure designed for the transducer must in practise be quantized into individual electrodes. This leads to interdigital-type transducers (IDTs) [19, 20].

Since the transducer displays the spatial period corresponding to $\left(k_{x, 0}, k_{y, 0}\right)$, the excited elastic wave has an equal periodicity. Therefore, the wave contains Fourier components for $k_{x, m}=m \omega s_{x}$ and $k_{y, m}=m \omega s_{x}$ where $m$ assumes integer values. The field is then represented as

$$
\mathbf{u}(x, z)=\sum_{m=-\infty}^{\infty} \sum_{n=1}^{4} A_{m, n} \mathbf{U}_{m, n} e^{i\left(k_{x, m} x+k_{y, m} y+k_{z, m, n} z\right)}
$$

Here, $m$ enumerates the different Fourier modes, while $n$ refers to the four different wave modes: L, FT, ST, and EM (an evanescent electromagnetic mode, see Ref. [21]), 
each of which may have a different $k_{z, m, n}$. Here $A_{m, n}$ are the complex-valued weights of each wave mode and $\mathbf{U}_{m, n}$ are their polarization vectors. This representation is analogous to the optical Rayleigh expansion [22] that is widely used in the analysis of diffractive gratings. We point out here that for a fixed $k_{x}$, there exist actually eight different modes. Four of these are discarded since they either propagate energy along positive $z$ or they grow exponentially along negative $z$ (the beam generated into the bulk is taken to propagate along $-z$ ), see Fig. 8. A straightforward and very useful algorithm for finding these modes has been published by Peach in Ref. [21].

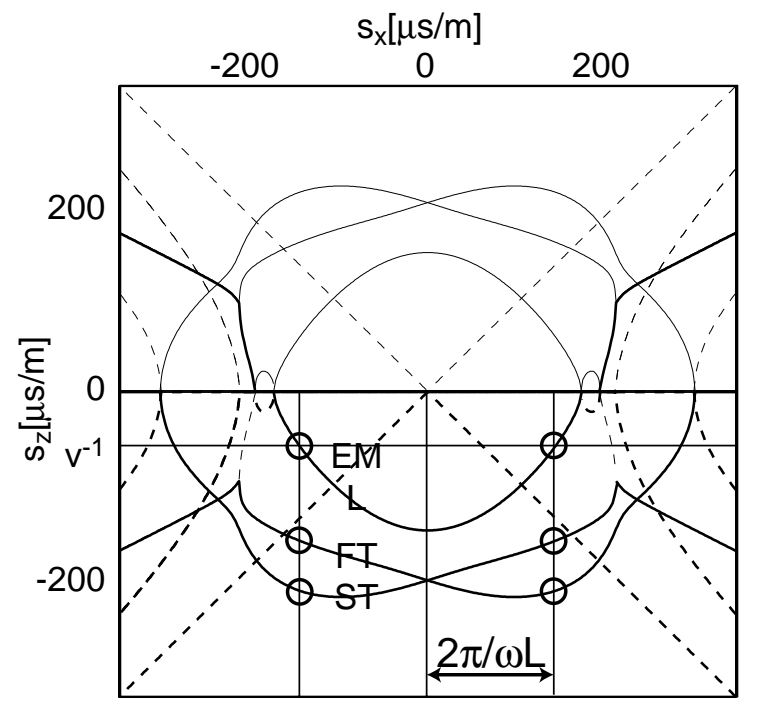

FIG. 8: Structure of the slowness surface in quartz and a schematic transducer layout for generating a continuouswave nondiffracting beam. Complex $s_{z}$ values as a function of $s_{x}$ for $s_{y}=0$. Here $\operatorname{Re}\left(s_{z}\right)$ are shown with solid lines and $\operatorname{Im}\left(s_{z}\right)$ with dashed lines. Thick lines propagate (or evanesce) along positive $z$ and thin lines along negative $z$. All generated modes are encircled and the entire beam is here taken to propagate along the direction of negative $z$.

Although the field expansion in Eq. (17) contains an infinite number of modes, the field is to be designed such that, ideally, only a minimul number of modes with real $s_{z}$ are actually generated. The most likely 'extra modes' to appear are those with $m=0$ and $m=-1$. The former always has three propagating modes but it is quite easily avoided by requiring that the average potential and the net charge on the surface remain zero. The latter has the same length for the surface wave vector as the desired mode and it has, subsequently, often a real $k_{z}$. If a transducer layout exhibits inversion symmetry, the $m= \pm 1$ modes are generated with equal weight, although the underlying anisotropy may still modify their relative amplitudes.

In the simplest of transducer schemes, a timeoscillating electric potential pattern is produced on a free surface of a piezoelectric crystal using (infinitely) thin metal electrodes. In this case, all three stress components along the surface normal must vanish for all the Fourier modes separately. There are four wave modes available (L, FT, ST and EM), and the condition of vanishing stresses can always be fulfilled by a suitable combination of them. Hence the field is expressed as

$\mathbf{u}(x, z)=\sum_{m=-\infty}^{\infty} \tilde{A}_{m} \sum_{n=1}^{4} w_{m, n} \mathbf{U}_{m, n} e^{i\left(k_{x, m} x+k_{y, m} y+k_{z, m, n} z\right)}$

where $w_{m, n}$ are weights such that the surface stresses of $\sum_{n=1}^{4} w_{m, n} \mathbf{U}_{m, n} e^{i\left(k_{x, m} x+k_{y, m} y\right)}$ vanish for each $m$. This combined mode may have an arbitrary amplitude but it requires a specific electric potential and charge distribution on the substrate surface. The transducer must be designed to provide these. Note that this scheme ignores all elastic properties of the transducer elements and it assumes a mechanically free interface between the crystal substrate and air. Transducers with electrodes of finite thickness can be analyzed numerically and the essential difference is that the resultant combination of L, FT, ST, and EM modes changes. This has been achieved, for instance, by Koskela et al. for surface-acoustic wave transducers, see Ref. [23].

We note that the generation of surface-acoustic waves (SAWs) using IDTs is well understood and they have a very important role in $\mathrm{RF}$ filter technologies. In SAW filters, however, the desired wave modes are always evanescent and the excitation of bulk-acoustic waves (BAWs) only leads to unwanted energy losses. IDTs have also been used for BAW excitation (see, for instance, Refs. $[24,25]$, and the brief discussion in the Appendix F of Ref. [26]), but they have not yet found important commercial applications. In the language of SAW technology, the transducers considered in this subsection are called leaky-SAW (LSAW) transducers that 'leak' wave energy into the bulk of the crystal. The difference is that, instead of minimizing this leakage, these transducers are optimized for the generation of certain bulk modes.

\section{B Aperture optimization}

Although, ideally, nondiffracting waves have an infinite beam length, the aperture size limits it to a finite value. In an isotropic medium, the wave emanating from the edge of a circular aperture, at the distance $R$ from the beam axis, moves to the beam axis at the angle $\zeta$, called the cone angle of the beam. It crosses the axis at $Z=R \cot \zeta$. A similar effect takes place in anisotropic media, except that there exists no uniform cone angle in this case. All directions have different plane waves (or several plane waves in the regions of internal diffraction) that carry energy at the different angles. Hence, each direction has an individual 'radius' of aperture $R(\varphi)=Z \tan \zeta(\varphi)$, where $\varphi$ is the azimuthal 

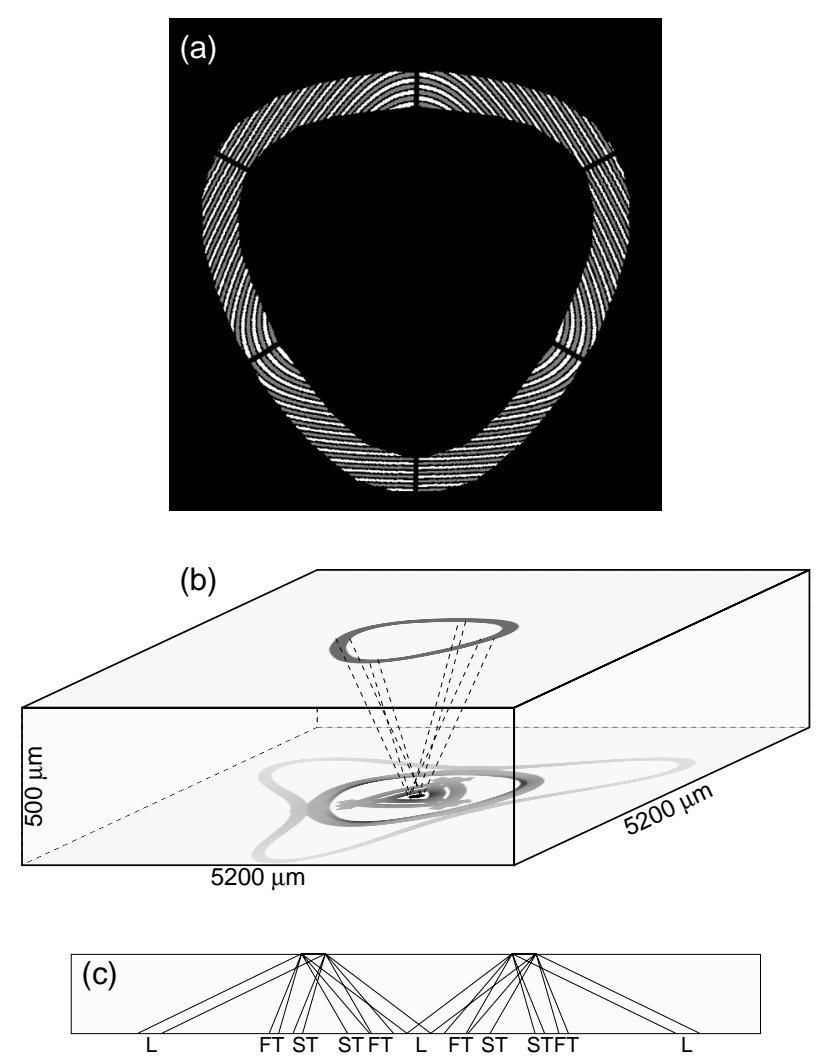

FIG. 9: (a) Sketch of a transducer for exciting an L-mode nondiffracting beam in quartz. Here white denotes the grounded electrodes, gray the driving electrodes, and the black areas are free substrate surface. Bondings of the electrodes must be supplied separately. (b) The entire radiation pattern of the transducer as observable on the opposite crystal surface. (c) Nondiffracting L-mode (in the centre) and spurious modes generated at the $(y=0)$ plane of the transducer. Each mode propagates in the direction specified by the associated group velocity.

angle on the crystal surface and $\zeta(\varphi)=\arctan \left(V_{r} / V_{z}\right)$ is the group-velocity angle for the plane wave entering from that direction.

In Fig. 9 there is illustrated a transducer that is designed to produce an L-mode beam in quartz. The interdigital transducer unavoidably produces one extra Lmode, two FT-modes, and two ST-modes. Therefore, the aperture is designed such that these do not overlap the intended nondiffracting beam in the center of the opposing crystal surface where the waves can be detected. The aperture is parametric in the sense that this may be scaled arbitrarily depending in the thickness of the quartz substrate. Note that it is independent of frequency that only affects the scaling of the transducer structure within. Propagation of the generated field modes is here considered within the limits of geometrical optics and all diffraction effects are discarded.

\section{Detection of NDWs}

The generation of a nondiffracting wave into a crystalline solid is the first part of the experimental research, while the detection of the wave is the second task. One way of detecting NDWs is the use of interferometric scanning on the back face of the crystal [28, 29]. This allows the detection of the surface-normal oscillation with an $x y$-resolution $\approx 1 \mu \mathrm{m}$ and amplitude threshold $\approx 0.1 \mathrm{~nm}$.

\section{CONCLUSIONS}

We have considered nondiffracting wave propagation in anisotropic crystals and analyzed the physical properties of nondiffracting waves based on their asymptotic representation. This has lead to a straightforward interpretation in terms of plane waves that have well-defined wave vectors and group velocities. The asymptotic representation also reveals phonon focusing, caustic, and internal diffraction effects that occur in anisotropic materials.

We have also considered the excitation of nondiffracting waves using an interdigital-type transducer that may be designed using the asymptotic properties of the specific beam form. Although similar devices (computergenerated holograms) have been widely used in optics, the presence of anisotropy and several acoustic modes lead to a much more complicated situation, and numerical modeling is required for the optimization of the local transducer structure.

Experimental research on anisotropic elastic wave propagation has recently benefitted from new imaging methods. The generation and detection of nondiffracting wave modes is a new challenge for experimental research; we hope that our results will stimulate further experiments.

\section{ACKNOWLEDGEMENTS}

JS is grateful to the Finnish Cultural Foundation, the Jenny and Antti Wihuri Foundation, and the Foundation of Technology (Finland) for financial support. We are grateful to Prof. A. R. Baghai-Wadji for pertinent comments on the manuscript. This research is also funded by the Academy of Finland through the Research Programme on Electronic Materials and Microsystems (EMMA).

\section{APPENDIX: OSCILLATORY INTEGRAL}

Consider an integral on a closed curve, parameterized by $\theta$, and given as [30]

$$
I=\int f(\theta) e^{i \alpha \phi(\theta)} d \theta
$$


where $\phi$ is real. The contribution of a small interval $\left[\theta_{0}-\epsilon, \theta_{0}+\epsilon\right]$ of the integral path, centered at $\theta_{0}$ is

$$
I_{\theta_{0}, \epsilon} \approx f\left(\theta_{0}\right) e^{i \alpha \phi\left(\theta_{0}\right)} \int_{-\epsilon}^{\epsilon} e^{i \alpha\left[\xi \phi^{\prime}\left(\theta_{0}\right)+\xi^{2} \phi^{\prime \prime}\left(\theta_{0}\right) / 2\right]} d \xi .
$$

Since the integral is dominated by $\xi=0$, it is highly oscillatory, except for $\phi^{\prime}=0$. If $\phi^{\prime}=0$, the value of the integral is approximately

$$
\int_{-\epsilon}^{\epsilon} e^{i \alpha \xi^{2} \phi^{\prime \prime} / 2} d \xi \approx \int_{-\infty}^{\infty} e^{i \alpha \xi^{2} \phi^{\prime \prime} / 2} d \xi=e^{ \pm i \pi / 4} \sqrt{\frac{2 \pi}{\alpha\left|\phi^{\prime \prime}\right|}}
$$

where \pm refers to the sign of $\phi^{\prime \prime}\left(\theta_{0}\right)$. We have changed the integration variable to $\xi=\sqrt{\frac{\pi}{\alpha\left|\phi^{\prime \prime}\right|}} t$. The integration limits have been extended from $[-\epsilon, \epsilon]$ to $[-\infty, \infty]$ since for sufficiently large $r$, the main contribution arises from an infinitesimal interval around the origin. If $\phi^{\prime} \neq 0$, the oscillating integrand averages to zero. Therefore, the total contribution of each $\phi^{\prime \prime} \neq 0$ is

$$
I \approx \sqrt{\frac{2 \pi}{\alpha\left|\phi^{\prime \prime}\left(\theta_{0}\right)\right|}} f\left(\theta_{0}\right) e^{i \alpha \phi\left(\theta_{0}\right)} e^{ \pm i \pi / 4} .
$$

This result is valid for a large enough parameter $\alpha$. It should be noted that a rapidly changing $f(\theta)$ or a low absolute value of $\left|\phi^{\prime \prime}(\theta)\right|$ requires a very large value of $\alpha$.

\section{REFERENCES}

[1] J. P. Wolfe, Imaging phonons: acoustics wave propagation in solids (Cambridge University Press, Cambridge, 1998).

[2] B. Taylor and H. J. Maris, and C. Elbaum, Phys. Rev. Lett. 23(8), 416 (1969); Phys. Rev. B 3(4), 1462 (1971).

[3] J. Durnin, J. J. Miceli, Jr., and J. H. Eberly, Phys. Rev. Lett. 58(15), 1499 (1987).

[4] J. Salo, J. Fagerholm, A. T. Friberg, and M. M. Salomaa, Phys. Rev. Lett. 83(6), 1171 (1999).

[5] J.-y. Lu and J. F. Greenleaf, IEEE Trans. Ultrason., Ferroelec., Freq. Contr. 39(1), 19 (1992).

[6] J. Fagerholm, A. T. Friberg, J. Huttunen, D. P. Morgan, and M. M. Salomaa, Phys. Rev. E 54(4), 4347 (1996).

[7] For geometrical caustics in optics see, for instance, J. F. Nye, Natural Focusing and Fine Structure of Light: Caustics and Wave Dislocations (IOP Publishing Ltd, London, 1999).

[8] M. R. Hauser, R. L. Weaver, and J. P. Wolfe, Phys. Rev. Lett. 68(17), 2604 (1992).

[9] L. D. Landau and E. M. Lifshitz, Theory of elasticity (Pergamon Press, 1986), 3rd ed.
[10] M. A. Auld, Acoustic fields and waves in solids, vol. 1 (John Wiley \& Sons, New York, 1973).

[11] J. Salo, J. Fagerholm, A. T. Friberg, and M. M. Salomaa, in IEEE Ultrason. Symp. Proc. (Institute of Electrical and Electronic Engineers, New York, 1998), pp. 1179-1182.

[12] J. Salo, J. Fagerholm, A. T. Friberg, and M. M. Salomaa, Phys. Rev. E 62(3), 4261 (2000).

[13] This is, in fact, similar to the presentation of an ordinary wave front, and leads to analogous treatment of phonon focusing and other anisotropic effects as used by H. J. Maris, Phys. Rev. B 28(12), 7033 (1983).

[14] I. S. Gradshteyn and I. M. Ryzhik, Table of Integrals, Series, and Products (Academic Press, Inc., New York, 1994), 5th ed.

[15] J. H. McLeod, J. Opt. Soc. Am. A 44(8), 591 (1954).

[16] F. V. Bunkin, V. V. Korobkin, Y. A. Kurinyi, L. Y. Polonskii, and L. N. Pyatnitskii, Sov. J. Quantum Electron. 13(2), 254 (1983).

[17] G. Scott and N. McArdle, Opt. Engineer. 31(12), 2640 (1992).

[18] J. Turunen, A. Vasara, and A. T. Friberg, Appl. Opt. 27(19), 3959 (1988).

[19] R. M. White and F. W. Voltmer, Appl. Phys. Lett. $7(12), 314$ (1965).

[20] R. F. Milsom, N. H. C. Reilly, and M. Redwood, IEEE Trans. Sonics and Ultrason. SU-24(3), 147 (1977).

[21] R. C. Peach, in IEEE Ultrason. Symp. Proc. (Institute of Electrical and Electronic Engineers, New York, 1995), pp. 221-225.

[22] R. Petit, ed., Electromagnetic theory of gratings (Springer-Verlag, Berlin, 1980).

[23] J. Koskela, V. P. Plessky, and M. M. Salomaa, IEEE Trans. Ultrason., Ferroelec., Freq. Contr. 46(4), 806 (1999).

[24] M. F. Lewis, Electron. Lett. 17(21), 819 (1981).

[25] M. A. Goodberlet and D. L. Lee, IEEE Trans. Ultrason., Ferroelec., Freq. Contr. SU-31(2), 67 (1984).

[26] D. P. Morgan, Surface-wave devices for signal processing (Elsevier, Amsterdam, 1985).

[27] A. R. Baghai-Wadji, in $14^{\text {th }}$ Annual Review of Progress in Applied Computational Electromagnetism (Monterey, CA, 1998), vol. 1, pp. 297-304.

[28] J. V. Knuuttila, P. T. Tikka, and M. M. Salomaa, Opt. Lett. 25(9), 613 (2000).

[29] J. V. Knuuttila, J. Saarinen, C. S. Hartmann, V. P. Plessky, and M. M. Salomaa, Electron. Lett. 37(16), 1055 (2001).

[30] For oscillatory integrals in the context of caustics, see, for instance, V. I. Arnold, Singularities of Caustics and Wave Fronts, Mathematics and Its Applications (Kluwer Academic Publishers, Dordrecht, 1990). 